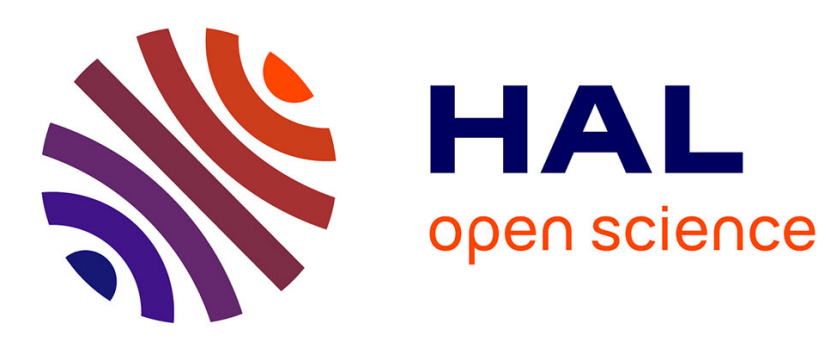

\title{
Core decomposition in Directed Networks: Kernelization and Strong Connectivity
}

Vincent Levorato

\section{To cite this version:}

Vincent Levorato. Core decomposition in Directed Networks: Kernelization and Strong Connectivity. Complex Networks, Mar 2014, Bologne, Italy. pp.129-140, 10.1007/978-3-319-05401-8_13 . hal00961165

\section{HAL Id: hal-00961165 https://hal.science/hal-00961165}

Submitted on 19 Mar 2014

HAL is a multi-disciplinary open access archive for the deposit and dissemination of scientific research documents, whether they are published or not. The documents may come from teaching and research institutions in France or abroad, or from public or private research centers.
L'archive ouverte pluridisciplinaire HAL, est destinée au dépôt et à la diffusion de documents scientifiques de niveau recherche, publiés ou non, émanant des établissements d'enseignement et de recherche français ou étrangers, des laboratoires publics ou privés. 


\title{
Core decomposition in Directed Networks: Kernelization and Strong Connectivity
}

\author{
Vincent Levorato $^{1,2}$ \\ 1 CESI, 959 rue de la Bergeresse, 45160 OLIVET, FR \\ 2 Université d'Orléans, LIFO, Bat. IIIA rue Léonard de Vinci, 45067 ORLEANS, FR \\ vlevoratodcesi.fr, vincent.levorato@univ-orleans.fr \\ Pre-print version
}

\begin{abstract}
In this paper, we propose a method allowing decomposition of directed networks into cores, which final objective is the detection of communities. We based our approach on the fact that a community should be composed of elements having communication in both directions. Therefore, we propose a method based on digraph kernelization and strongly $p$-connected components. By identifying cores, one can use based-centers clustering methods to generate full communities. Some experiments have been made on three real-world networks, and have been evaluated using the V-Measure, allowing a more precise analysis through its two sub-measures: homogeneity and completeness. Our work proposes different directions about the use of kernelization into structure analysis, and strong connectivity concept as an alternative to modularity optimization.
\end{abstract}

Keywords: cores;communities detection;directed networks;kernelization;strong connectivity;graph theory;clustering;

\section{Introduction}

Complex networks appear in many applications, including social networks analysis on the Web, which is a topical research subject. These networks carry non-trivial topological properties that characterize their connectivity, and affect the dynamics of their behaviors. The analysis of complex networks often leads to the analysis of the roles of elements, or groups of elements, composing a network. Communities detection belongs to this research field, and can be very useful to better understand how networks are structured. In this article, we focus on the problem of finding communities in networks, and more specifically finding cores in directed networks. Dealing with methods of community detection for directed networks is a difficult task, and few methods exist compared to methods used in the undirected case. Here are some of the most known works in the literature [9, 18, 14] dedicated to directed networks, or which can be adapted to work with directiveness: Clauset et al. method [5], CFinder based on the Clique Percolation Method (CPM) [23], Louvain method [3], InfoMap [26], Simulated Annealing for modularity [11], Wu-Huberman method [34], MarkovCluster algorithm [32], Multistep Greedy algorithm [27] and EM method (Expectation-Maximization) [20]. More 
recently, others methods concerning directed networks have been proposed, with more or less good results [35, 16, 15].

Generally, these methods are most of the time designed for undirected networks, and adjusted to work in the directed case: they are not initially dedicated to the directed case. There is also a significant amount of methods using modularity optimization. However, this kind of approach has its limits, and can "miss important substructures of a network" [10]. Some recent work discuss about reciprocated interaction [4], that two people should communicate in both directions, the first person expecting messages from the second person, and vice versa. Our approach is based on this simple idea: in a directed network, a community should be composed of nodes which can communicate with every nodes in the community, in both directions. Interesting results in our previous exploratory work [21] encourage us to continue in this direction. Usually represented by graphs in undirected networks, this kind of representation can be modeled by the connected component concept, and more restrictively by the clique concept. Except that for the directed case, it can be represented by the concept of strongly connected component. Finding these components should be equivalent to find cores to which other elements of the network will be assigned. This work gives the key concepts of this approach, focusing on the cores finding.

Our paper is structured as such: the first section gives the definitions of graph theory and formal concepts needed to understand our method. Then, the second part exposes the different steps of our approach, followed by some experimental results on real networks. The paper ends by a conclusion which opens discussion on future work directions.

\section{Graph Theory Notions}

\subsection{Graph definitions}

In this article, we consider only directed graphs (also noted digraph). We give here a short reminder of graph theory notions. Formally, a digraph $G=(V, A)$ is the pair composed of [2]:

- a set $V=\left\{x_{1}, x_{2}, \ldots, x_{n}\right\}$ named vertices or nodes.

- a family $A=\left(a_{1}, a_{2}, \ldots, a_{n}\right)$ of elements of the Cartesian product $V \times V=$ $\{(x, y) / x \in V, y \in V\}$ named arcs.

The amount of vertices is noted $n$ (also noted $|V(G)|$ ) and the amount of arcs is noted $m$ (also noted $|A(G)|$ ).

A path $P$ is composed of $k$ arcs such as $P=\left(a_{1}, a_{2}, \ldots, a_{i}, \ldots, a_{k}\right)$ where for every arc $a_{i}$ the terminal end coincides with the initial end of $a_{i+1}$. Several equivalent notations can be used: $P=\left(\left(x_{1}, x_{2}\right),\left(x_{2}, x_{3}\right), \ldots\right)=\left[x_{1}, x_{2}, \ldots, x_{k}, x_{k+1}\right]=P\left[x_{1}, x_{k+1}\right]$.

A chain is, like a path, an alternating sequence of vertices and edges, where an edge is an arc without orientation.

A circuit is a path such that the first node of the path corresponds to the last. It can be viewed as an oriented cycle. 


\subsection{Connected Components}

Here are the different types of connected components we could have in a directed graph [13]:

- a weakly connected component $W C C$ of a digraph is a subgraph where: $\forall x, y \in$ $W C C$, there is a chain between $x$ and $y$.

- an unilaterally connected component $U C C$ of a digraph is a subgraph where: $\forall x, y \in U C C$, there is a path between $x$ and $y$ OR there is a path between $y$ and $x$.

- a strongly connected component $S C C$ of a digraph is a subgraph where: $\forall x, y \in$ $S C C$, there is a path between $x$ and $y$ AND there is a path between $y$ and $x$.

To discover cores in a network, we use a special case of strongly connected component named strongly p-connected component by [33] which is related to l-edgeconnectivity [7] (we use p-connected notation instead of $n$-connected to avoid confusion):

- a strongly p-connected component $p$-SCC of a digraph is a subgraph where: $\forall x, y \in$ $p$-SCC, there is a path of length $p$ or less between $x$ and $y$, and there is a path of length $p$ or less between $y$ and $x$, with $p \geq 2$.

\section{Core detection}

\subsection{Related work}

Finding cores in order to find communities is a method that can be related to pattern identification [14]. This consists in finding maximal subsets which implies separation between them. Clique finding is one of these methods, but is also very restrictive, because each node must have a direct connection to other nodes. This approach has been relaxed by the $n$-clique definition where each node is connected to others by at least one path which length is at most $n$, but that can be outside of the $n$-clique. The $n$-clan concept, by adding a constraint on the diameter of a $n$-clique that should not be greater than $n$, fixes the connectedness issue of the $n$-clique [22]. Some concepts in the directed case exist such as the $f$-groups (maximal subsets of weakly and strongly transitive triads, like 3-clique or triangle) [12], and directed $k$-clique [24]. Our approach is related to these works, but we don't put strong constraint on the size (triads), and we don't want to avoid circuits (directed $k$-clique), as it is specifically the configuration we are looking for: strongly $p$-connected components.

\subsection{Searching for cores using p-SCC concept}

Our community definition refers to a group containing elements that can communicate with all other elements of the group. In digraphs, this idea is formalized by the concept of strongly connected components (SCC). Searching for SCCs in a digraph is equivalent to search for circuits, and Tarjan based his algorithm on this idea [29], which returns maximal strongly connected subgraphs. To our knowledge, no work has considered the 
SCCs in the case of researching communities. By simply applying Tarjan's algorithm on directed graph generated through LFR benchmark [17], some communities can be found, but SCCs are often oversized. To refine the process, our approach proposes to find $p$-SCCs (fig. 1). The problem is that in a digraph, the number of circuits may be exponential in the number of vertices [30]. Therefore, processing all circuits of a graph is not relevant, especially if the graph has a significant number of nodes like in large real-world networks.

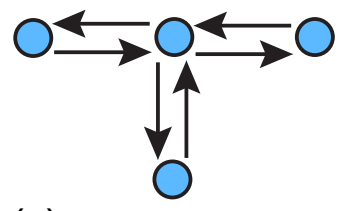

(a)

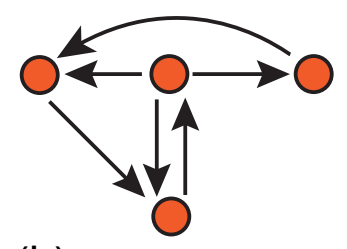

(b)

Fig. 1. Examples of $p$-SCCs: $(a)$ : nodes are connected by paths of length at most 2 (2-SCC) $(b)$ : nodes are connected by paths of length at most 3 (3-SCC).

To be able to process large graph, our method finds $p$-SCCs by starting from a given node $s$. Starting from this node, finding $p$-SCC also means searching for circuits, but circuits with a given size. Trivially, the length of the path $p$ is bounded by the length of the circuits found into the $p$-SCC :

$$
p \leq 2 \times(c-1)
$$

where $c$ is the size of circuits we are searching for.

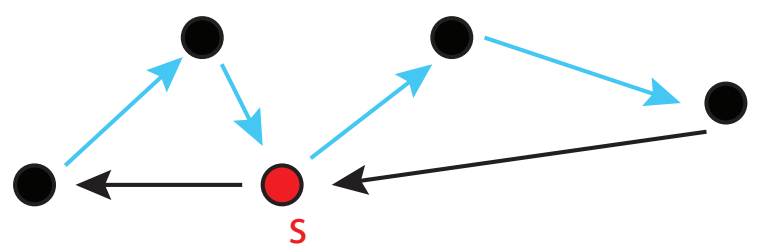

Fig. 2. Searching $p$-SCC is similar to search circuits from a starting node $s$. In this case, searching for 4-SCC means searching for circuits of length at most 3 . The highlighted path length is 4 , the maximal path length which can be found. 
For instance, searching for circuits of length 3 starting from a node means searching for at most 4-SCCs (fig. 2). We propose an algorithm which returns a $p$-SCC starting from a given node (alg. 11). As the algorithm is searching for circuits, and considering that $p$ parameter sets the circuit length, we can only find $p$-SCCs with $p$ being an even number. It is written in a non-recursive way, but time complexity should be approximatively the same as Tarjan's algorithm, which is $O(n+m)$, with two differences: we don't always need to pass through every arc (depends on path length), but we should pass through nodes several times.

Input: $G$ : digraph, $s$ :starting node,

$p$ :path length (even integer)

Data: astack: stack of arcs, vpath: stack of nodes,

$c$ : integer (circuit size)

Remark: Aout $(k)$ represents set of outing arcs of the node $k$.

$\operatorname{source}(a)$ represents the source node of the arc $a$.

$\operatorname{dest}(a)$ represents the destination node of the $\operatorname{arc} a$.

Result: $C$ :set of nodes ( $p$-SCC)

$C \leftarrow \emptyset ; C \leftarrow C \cup\{s\}$;

vpath.push $(s) ; c \leftarrow \frac{(p+2)}{2}$;

foreach $a$ in Aout(s) do

| astack.push(a);

end

while astack $\neq \emptyset$ do

$a \leftarrow$ astack.pop ()$;$

$w \leftarrow$ vpath.peek ()$;$

if $\operatorname{source}(a) \neq w$ then

while $\operatorname{source}(a) \neq w$ do

$w \leftarrow$ vpath.pop ()$;$

end

vpath.push $(w)$;

end

$z \leftarrow \operatorname{dest}(a)$

if $z=s$ then

$C \leftarrow C \cup$ vpath;

end

else

if $\mid$ vpath $\mid<c$ then

foreach $b$ in $\operatorname{Aout}(z)$ do

| astack.push(b);

end

vpath.push(z);

end

end

end

return $C$;

Algorithm 1: Algorithm extracting $p$-SCC. 


\subsection{Digraph kernelization}

In the previous part, we explained the general idea of our approach, finding communities cores by searching $p$-SCC, which means exploring a digraph in order to find circuits. Before describing the whole method based on core detection, we show how we can optimize the search for $p$-SCCs by "cleaning" the digraph, meaning excluding nodes and arcs which should never belong to a circuit: this brings us to the notion of graph kernelization. We are interested in the kernelization which is used in the FVS problem (Feedback Vertex Set) [31]. Its purpose is to find a set of nodes which gives a graph without cycles if this set of nodes (and their adjacent edges) is removed from the graph. The general interest of the kernelization process is the reduction of the size of the input given to an algorithm which is not polynomial in time (in most cases): the graph is being "compressed". As we work in the directed case, we used the kernelization technique applied to the directed FVS [8], following the four first rules of the method. The two first rules imply a simple digraph (no self-loop, no multiple arcs), the third one removes isolated nodes (degree equal to zero), and the last one removes the chained nodes, by removing nodes from the digraph while it contains nodes with only one outgoing or incoming arc. The experiment part shows that the kernelization operation can be very effective on real-world networks.

\section{Digraph cores decomposition method}

This section describes our method for core detection in directed networks. Let use the following notations: $G$ is the input digraph (network), and $\mathcal{K}$ is the set of output cores. The method follows these steps, considering a given $p$ :

1. Kernelize $G$.

2. For each node of $G$ as the starting node, process $p$-SCC.

3. Sort $p$-SCCs by size. Starting from the biggest one, put them one by one in $\mathcal{K}$ if it doesn't intersect existing cores already inserted into $\mathcal{K}$. In case of cores having the same size, take the most connected one (biggest amount of arcs).

4. (optionnal) Remove $p$-SCCs with size inferior to a given threshold $K_{m i n}$.

Illustration: The figure 3 gives an illustration of our method, step by step, with $p=4$ (meaning we search for circuits of length at most 3). Let take a digraph (a), and apply the first step which is kernelization (b). Some nodes are ignored, and won't be considered. The second step processes 4 -SCCs, node by node: in the example (c), only five iterations are represented (nodes with labels $4,5,8,10,13$ ), and for each node, a 4-SCC is computed, which can be the same for several nodes (nodes 5 and 8 produce the same 4-SCC, same thing for nodes 4 and 13). The last step (d) extracts the biggest and non-intersecting 4-SCCs giving the final result with 3 cores.

This method returns a set of cores which can be used to cluster the rest of the network to have a complete clustering. As some clustering methods like $k$-means algorithm, the number of communities is set by the number of cores. In this article, as we don't focus on clustering methods, our experiments use a simple aggregative method like center-based clustering methods, and assign each node to the community having 


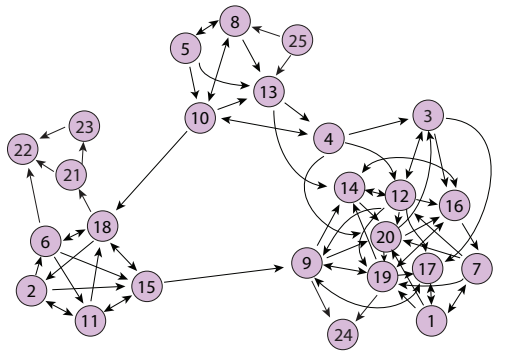

(a) Input digraph

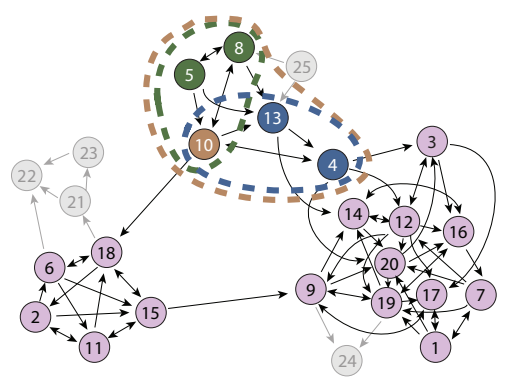

(c) Processing the 4-SCCs

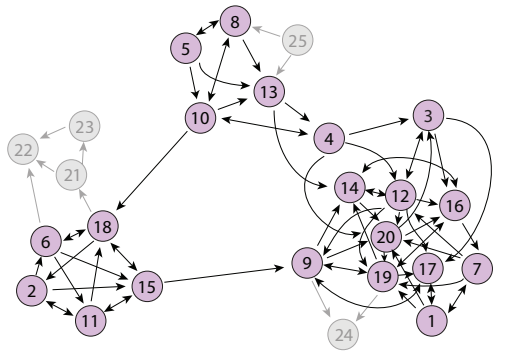

(b) Kernelization

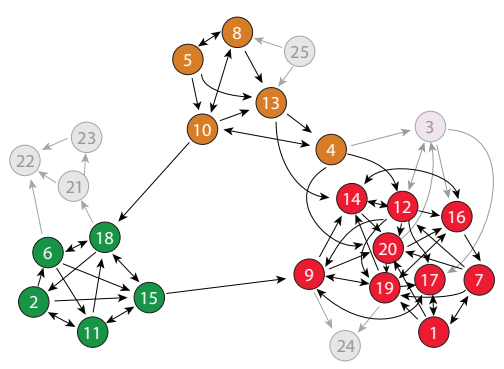

(d) Cores result

Fig. 3. Illustration of the decomposition method of a digraph into cores.

the nearest core. The distance corresponds to the chain length from node to core (at this point, link orientation is ignored). At each step, the cores absorb their nearest neighbors, and the process continues until all the nodes belong to a group. If a node can be absorbed by more than one community, meaning the distance between several cores is equal, it will be absorbed by the community sharing the biggest amount of edges with it. The $K_{\min }$ value can be useful to avoid too small cores that shouldn't be considered.

\section{Experiments}

Validating communities structures corresponds to validate a clustering method. The difficulty is to find an objective measure of quality of clusters. For our experiments, we use V-Measure which is an alternative to F-Measure, and Normalized Mutual Information from the information theory field to compare the clustering obtained by our method to the reference classes. We based our experiments on real data networks, as some experiments have already been done on generated networks (LFR Benchmarks [17]) in our previous work with good results [21]. Moreover, there is an issue with the LFR Benchmark, as it produces graphs already kernelized, which puts a strong constraint on generated graphs. On the contrary, in the experiment part, we observe that the real-world networks we used are strongly kernelizable. 


\subsection{Clustering Evaluation}

The entropy notion is used to express the used measures, and is noted as follow, with $X$ and $Y$ two discrete random variables: $H(X)$ and $H(Y)$ for the marginal entropies, $H(X \mid Y)$ and $H(Y \mid X)$ for the conditional entropies, $H(X, Y)$ for the joint entropy. Measures give results between 0 (worst matching) and 1 (best matching). Here are the two evaluation measures definitions:

- V-Measure [25] is an entropy based-evaluation measure, composed of two concepts: completeness and homogeneity. The V-Measure corresponds to the harmonic mean of these two concepts, just like F-Measure with precision and recall. With $C$ a set of classes (reference), $K$ a set of clusters (unsupervised method), the homogeneity is defined as:

$$
h= \begin{cases}1 & \text { if } H(C, K)=0 \\ 1-\frac{H(C \mid K)}{H(C)} & \text { else }\end{cases}
$$

and the completeness is defined as:

$$
c= \begin{cases}1 & \text { if } H(K, C)=0 \\ 1-\frac{H(K \mid C)}{H(K)} & \text { else }\end{cases}
$$

A clustering result satisfies homogeneity if all of its clusters contain only elements which are members of a single class, and a clustering result satisfies completeness if all the elements that are members of a given class are elements of the same cluster. The V-Measure is based on homogeneity and completeness scores such as:

$$
V_{\beta}=\frac{(1+\beta) \cdot h \cdot c}{(\beta \cdot h)+c)}
$$

with a $\beta$ parameter which can be used to weight homogeneity and completeness scores.

This measure solves the problem of other measures like F-Measure which only consider the contributions from the clusters which match a target class, and has sub-measures useful to evaluate precisely the quality of a clustering solution. In our experiments, we set $\beta=1$ (balanced weights).

- Normalized Mutual Information (NMI) [6] Mutual information is a measure used in information theory domain, giving the amount of information that one random variable contains about another. The entropy reflects the self-information of a random variable. Mutual information can be can be summarized in a measure of the distance between two probability distributions. This measure is defined between the cluster assignments $K$ and a pre-existing labeling set of classes $C$ normalized by:

$$
N M I(K, C)=\frac{I(K, C)}{\sqrt{H(K) H(C)}}
$$


with $I(K, C)$ the mutual information of $K$ and $C$ such that:

$$
I(K, C)=H(K)-H(K \mid C)
$$

\subsection{Results}

In order to test our approach, we used directed network datasets already known in the literature [1, 28]. Three networks have been used: a political blog network about US politics, and two citation networks (Cora and Citeseer) (see tab. 11. Our method have been applied on the biggest connected component (WCC) to avoid noisy results. Several characteristics of the network are given like density, degree information, communities maximum and minimum sizes, but also the mixing parameter $\mu[18]$ and the directed modularity $Q_{d}$ [20].

\begin{tabular}{|c|c|c|c|}
\hline Directed Network & Political Blog & Cora & Citeseer \\
\hline$|V|$ & 1,222 & 2,485 & 2,120 \\
\hline$|A|$ & 19,024 & 5,209 & 3,768 \\
\hline Classes & 2 & 7 & 6 \\
\hline Density & $1.27 \%$ & $0.08 \%$ & $0.08 \%$ \\
\hline Degrees & $k_{\text {mean }}=31$ & $k_{\text {mean }}=4$ & $k_{\text {mean }}=4$ \\
& $k_{\text {min }}=1$ & $k_{\text {min }}=1$ & $k_{\text {min }}=1$ \\
& $k_{\text {max }}=467$ & $k_{\text {max }}=169$ & $k_{\text {max }}=100$ \\
\hline Communities size & $|C|_{\text {min }}=588$ & $|C|_{\text {min }}=131$ & $|C|_{\text {min }}=115$ \\
& $|C|_{\text {max }}=636$ & $|C|_{\text {max }}=726$ & $|C|_{\text {max }}=532$ \\
\hline$\mu$ & 0.09 & 0.18 & 0.28 \\
\hline$Q_{d}$ & 0.41 & 0.63 & 0.51 \\
\hline
\end{tabular}

Table 1. Network datasets.

The mixing parameter is used by Lancichinetti and al. to generate datasets of networks. It represents the fraction of all links in a particular community that end outside this community. More its value increases, less the communities are well defined and less easily detectable. Network modularity is defined as the fraction of all links that lie within communities minus the expected value of the same quantity in a network in which nodes have the same degree but with links placed randomly. It gives an idea on the "good" separation of communities.

For each network, we seek for cores, and study the results using only V-measure. Then we focus on the communities result with both NMI and V-Measure.

Core decomposition The quality of the core depends on the kernelization process, as graph compression is highly different from a network to another (tab. 22. In tab. 3 , we compare the obtained cores with the reference classes, using the $p$ parameter which corresponds to the path length of a $p$-SCC, and the $K_{\min }$ parameter which is the minimum core size (only relevant results are shown). In our experiments, the use of the minimum 
core size has been relevant. In most cases, cores have a good completeness score, meaning that we succeed in having nodes which belong to a single class in only one core. On the other hand, the homogeneity score tends to be better when the threshold of the minimum core size is increased (Political Blog and Cora networks), having nodes of a same core belonging to a single class. The interpretation that can be made from these results is that the more the graph is compressed, the less the $K_{\min }$ gets an high value. When too many nodes are available to build cores, the $K_{\min }$ threshold has to be high to remove some eventual noise, giving less nodes usable in the core creation (illustration fig. 4). In the results of tab. 3, we first consider completeness value, and then the homogeneity value. We give more importance to the completeness score, as it gives better results in the final process communities detection.

\begin{tabular}{|c|c|c|c|}
\hline Directed Network & Political Blog & Cora & Citeseer \\
\hline$|V|_{K}$ & 811 & 399 & 69 \\
\hline$|A|_{K}$ & 15,833 & 786 & 97 \\
\hline Compression rate (nodes) & $33 \%$ & $84 \%$ & $97 \%$ \\
\hline
\end{tabular}

Table 2. Network kernel sizes.

Communities detection Using an aggregative method, the cores first absorb nodes which are in the kernel but not in the cores, giving pre-built communities. Then, the nodes outside the kernel are absorbed by the pre-built communities to give a final clustering of communities. Clusters are not strongly connected, but unilaterally connected. The results in tab. 4 show that even with a naive method of clustering, the communities structures remain "acceptable". The cores used in the final clustering process are the cores having the best completeness scores in the core decomposition operation. Compared to InfoMap algorithm (tab. 5), which stays the method with best results obtained in directed networks [18], our results look pretty similar, with best performance on the Political Blogs network. Infomap also tends to subdivide too much the networks, finding 30 to 60 times more communities than expected. Observing the results, we can make the assumption that the compression of graphs impacts the quality of cores, and therefore the detection of communities. With a small amount of nodes in the kernel, the choice to make between the nodes to build the cores is important, as it determines the final process of communities detection. Also, having only big cores means setting a too high $K_{\min }$ threshold value, which can have a negative impact on the cores detection, and some communities cannot be found in the process. For instance, in fig. 5 , the central community has been found by our method using the parameters $p=4, K_{\min }=2$. If we set $K_{\text {min }}=3$, cores of size 2 are excluded, and this central community is not detected. 
(a) Political Blog

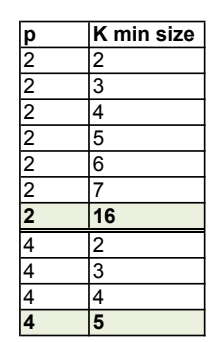

\begin{tabular}{|l|l|l|l|l|}
\hline \multicolumn{3}{|c|}{ V-Measure } & \multicolumn{2}{l}{} \\
\hline $\mathbf{h}$ & $\mathbf{c}$ & $\mathbf{V}$ & Nb Nodes & Nb Cores \\
\hline 0.35316 & 0.95295 & 0.51534 & 329 & 20 \\
\hline 0.40471 & 0.94876 & 0.56739 & 308 & 13 \\
\hline 0.44344 & 0.94601 & 0.60383 & 296 & 10 \\
\hline 0.52053 & 0.96137 & 0.67538 & 281 & 7 \\
\hline 0.59364 & 0.97468 & 0.73787 & 269 & 5 \\
\hline 0.7381 & $\mathbf{1 . 0}$ & 0.84932 & 255 & 3 \\
\hline $\mathbf{1 . 0}$ & $\mathbf{1 . 0}$ & 1.0 & 239 & 2 \\
\hline \hline 0.60926 & 0.98967 & 0.75421 & 401 & 12 \\
\hline 0.79398 & 0.98896 & 0.88081 & 380 & 5 \\
\hline 0.90979 & $\mathbf{1 . 0}$ & 0.95276 & 372 & 3 \\
\hline $\mathbf{1 . 0}$ & $\mathbf{1 . 0}$ & 1.0 & 367 & 2 \\
\hline
\end{tabular}

(b) Cora

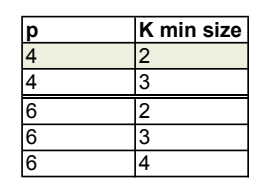

\begin{tabular}{|l|l|l|l|l|}
\hline \multicolumn{3}{|c|}{ V-Measure } & \multicolumn{2}{c}{} \\
\cline { 1 - 3 } $\mathbf{h}$ & $\mathbf{c}$ & $\mathbf{V}$ & Nb Nodes & Nb Cores \\
\hline $\mathbf{0 . 4 1 0 1 9}$ & $\mathbf{0 . 9 4 1 2}$ & 0.57137 & 98 & 28 \\
\hline 0.57836 & 0.9352 & 0.71471 & 47 & 11 \\
\hline \hline 0.41481 & 0.93729 & 0.5751 & 103 & 28 \\
\hline 0.58141 & 0.92778 & 0.71485 & 52 & 11 \\
\hline 0.70183 & 0.92268 & 0.79724 & 32 & 6 \\
\hline
\end{tabular}

(c) Citeseer

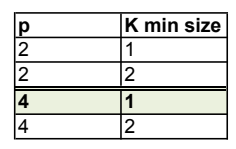

\begin{tabular}{|l|l|l|r|l|}
\hline \multicolumn{3}{|c|}{ V-Measure } & \multicolumn{1}{|l|}{} \\
\hline $\mathbf{h}$ & $\mathbf{c}$ & $\mathbf{V}$ & Nb Nodes & Nb Cores \\
\hline 0.49039 & 0.93415 & 0.64315 & 54 & 26 \\
\hline 0.19087 & 0.29364 & 0.23136 & 6 & 2 \\
\hline $\mathbf{0 . 4 8 9 9 4}$ & 0.93454 & 0.64285 & 58 & 26 \\
\hline 0.5907 & 0.77251 & 0.66948 & 12 & 3 \\
\hline
\end{tabular}

Table 3. Cores detection on real-world networks.

\begin{tabular}{|c|c|c|c|c|}
\hline Network & \multicolumn{4}{|c|}{ Measures } \\
\hline & $\mathrm{h}$ & c & $\mathbf{V}$ & NMI \\
\hline Political Blog & 0.70385 & 0.69929 & 0.70156 & 0.70116 \\
\hline Cora & 0.35335 & 0.46349 & 0.40099 & 0.40469 \\
\hline Citeseer & 0.28162 & 0.38734 & 0.32613 & 0.32742 \\
\hline
\end{tabular}

\begin{tabular}{|l|}
\hline $\begin{array}{c}\text { Amount of } \\
\text { Communities }\end{array}$ \\
\hline 2 \\
\hline 28 \\
\hline 26 \\
\hline
\end{tabular}

Table 4. Real-world networks communities detection results based on core decomposition.

\begin{tabular}{|l|l|l|l|l|}
\hline \multicolumn{1}{|c|}{ Network } & \multicolumn{4}{|c|}{ Measures } \\
& \multicolumn{4}{|c|}{} \\
\hline & h & c & V & NMI \\
\hline Political Blog & 0.281 & 0.759 & 0.410 & 0.462 \\
\hline Cora & 0.259 & 0.833 & 0.395 & 0.465 \\
\hline Citeseer & 0.228 & 0.721 & 0.347 & 0.404 \\
\hline
\end{tabular}

\begin{tabular}{|l|}
\hline $\begin{array}{c}\text { Amount of } \\
\text { Communities }\end{array}$ \\
\hline 56 \\
\hline 438 \\
\hline 311 \\
\hline
\end{tabular}

Table 5. Real-world networks communities detection results obtained by InfoMap. 


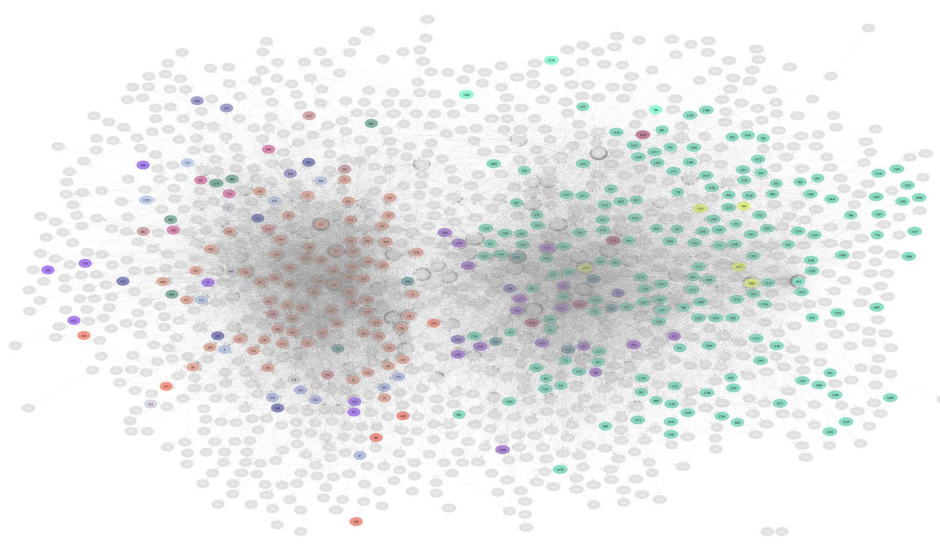

(a) Bad-defined cores (number of cores $=20$ )

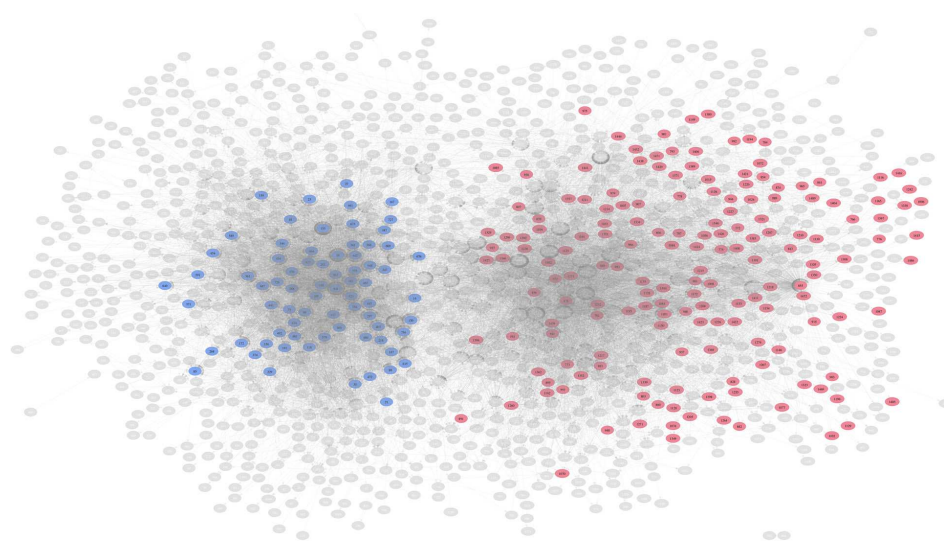

(b) Well-defined cores (number of cores $=2$ )

Fig. 4. Illustration of core decomposition in the Political Blog network.

\section{Conclusion}

In this article, we focused on an approach dedicated to directed networks, and we gave a method allowing the decomposition of these networks into cores. These cores can be used by any clustering method based on centers to detect communities. Our various contributions can be presented as follows:

- Using the strongly $p$-connected components is the key of our approach, which has not been used in any scientific work in order to detect cores or communities in directed networks. Moreover, we provide a simple and efficient algorithm to generate these $p$-SCCs in a digraph. This approach can be classified in the pattern identification category that we can find in some method classification, while being flexible enough. 


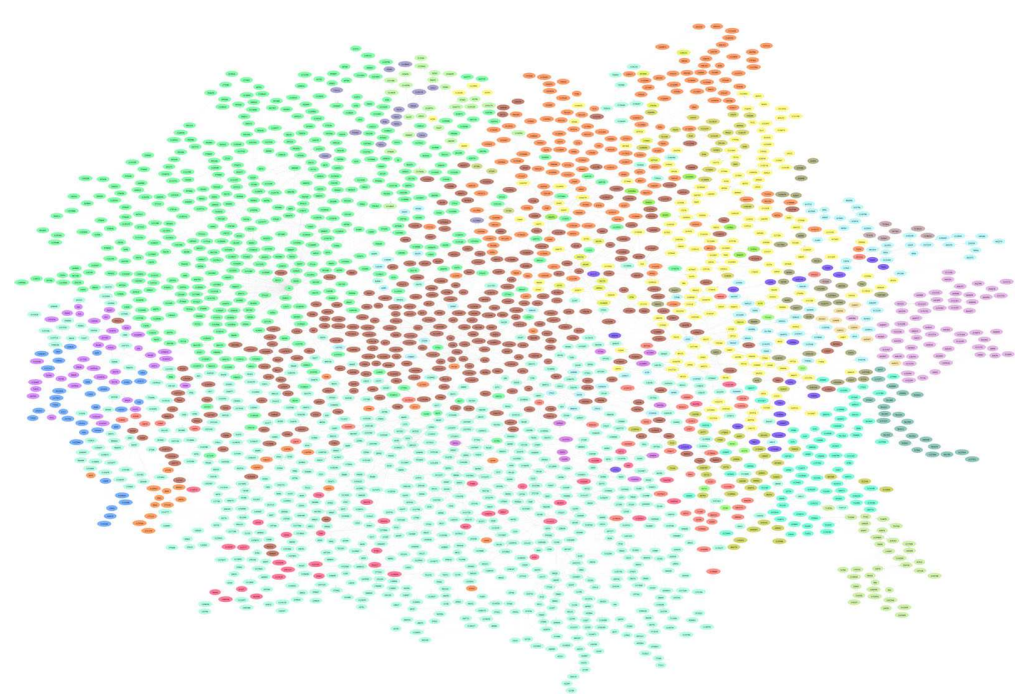

(a) Clustering using an aggregative method based on core decomposition.

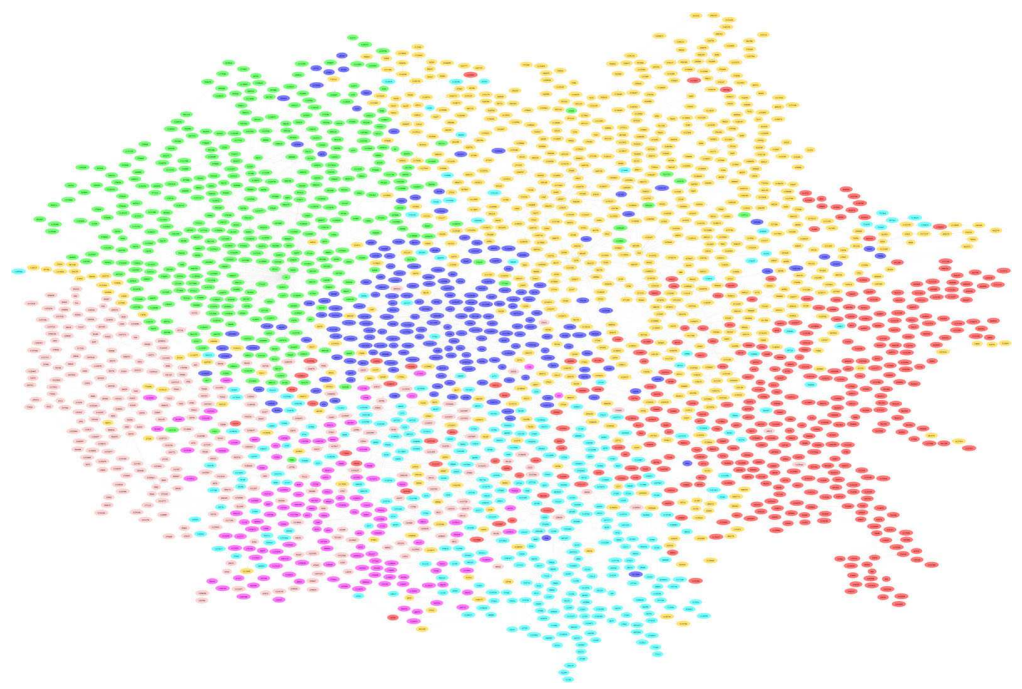

(b) Reference classes.

Fig. 5. Communities detection comparison on the Cora citation network ( $p=4, K_{\min }=2$ ). 
- The interest of using kernelization process has been highlighted : it reduces the core detection process, and can give some information on the network structure. The hypothesis of having the compression rate of a digraph correlated to the mixing parameter has to be taken into account, and more experiments on real-world networks have to be done to verify this intuition.

- During our experiments, we obtained some encouraging results on the network datasets we used. An important thing about these results is that we didn't take into account the modularity concept in our approach. As a large part of the communities detection algorithms are dedicated to modularity optimization [14], we want to stress the point that we can have interesting results in communities detection without this concept, which some limits are already known even in the undirected case [19].

Several options can be considered for the continuation of this work. As we said, we have to apply our method to others real-world datasets. We should also study how to increase the quality of the core detection, and it could be interesting to have the possibility to automatically fix the $K_{\min }$ threshold value. Testing other based-centers clustering methods should be done too. Also, the case of overlapping communities should be considered, as our approach could be quickly adaptable with $p$-SCCs which naturally overlap each other. In our opinion, our work points out that no clear or unanimous consensus about the definition of communities exists, and provides a new point of view on the detection of communities into directed networks, being omnipresent in the Web nowadays.

\section{Acknowledgments}

We would like to thank Pr. Ioan Todinca, Mathieu Liedloff, Anthony Perez and the GAMoC team of the LIFO at University of Orléans (FR) for their listening and advices. Special thanks to Guillaume Cleuziou from the Machine Learning team.

\section{References}

[1] Lada A. Adamic and Natalie Glance. The political blogosphere and the 2004 u.s. election: divided they blog. In Proceedings of the 3rd international workshop on Link discovery, LinkKDD '05, pages 36-43, New York, NY, USA, 2005. ACM.

[2] Claude Berge. Graphes et Hypergraphes. Dunod, Paris, 1970.

[3] Vincent D. Blondel, Jean-Loup Guillaume, Renaud Lambiotte, and Etienne Lefebvre. Fast unfolding of communities in large networks. Journal of Statistical Mechanics, Volume 2008(10), 2008.

[4] Justin Cheng, Daniel Mauricio Romero, Brendan Meeder, and Jon M. Kleinberg. Predicting reciprocity in social networks. In SocialCom/PASSAT, pages 49-56, 2011.

[5] Aaron Clauset, Mark E. J. Newman, and Cristopher Moore. Finding community structure in very large networks. Phys. Rev. E, 70(6):066111, 2004.

[6] Leon Danon, Albert Díaz-Guilera, Jordi Duch, and Alex Arenas. Comparing community structure identification. Journal of Statistical Mechanics: Theory and Experiment, 2005(9):P09008-09008, 2005. 
[7] Reinhard Diestel. Graph Theory. Springer-Verlag Berlin, 4th revised edition edition, July 2010.

[8] Rudolf Fleischer, Xi Wu, and Liwei Yuan. Experimental study of fpt algorithms for the directed feedback vertex set problem. In Amos Fiat and Peter Sanders, editors, Algorithms - ESA 2009, volume 5757 of Lecture Notes in Computer Science, pages 611-622. Springer Berlin Heidelberg, 2009.

[9] Santo Fortunato. Community detection in graphs. Physics Reports, 486(3-5):74-174, February 2010.

[10] Santo Fortunato and Marc Barthélemy. Resolution limit in community detection. Proceedings of the National Academy of Science, 104(1):36-41, 2006.

[11] Roger Guimerà and Luís A. Nunes Amaral. Functional cartography of complex metabolic networks. Nature, 433:895-900, 2005.

[12] Robert A. Hanneman and Mark Riddle. Introduction to social network methods. University of California, Riverside, Riverside, CA, 2005.

[13] Frank Harary. Graph Theory. Addison-Wesley, Reading, 1969.

[14] Vincent Labatut and Jean-Michel Balasque. Detection and interpretation of communities in complex networks: Practical methods and application. Computational Social Networks: Tools, Perspectives and Applications, pages 81-113, 2012.

[15] Darong Lai, Hongtao Lu, and Christine Nardini. Finding communities in directed networks by pagerank random walk induced network embedding. Physica A: Statistical Mechanics and its Applications, 389(12):2443 - 2454, 2010.

[16] Renaud Lambiotte, Roberta Sinatra, Jean-Charles Delvenne, T. S. Evans, Mauricio Barahona, and Vito Latora. Flow graphs: Interweaving dynamics and structure. Phys. Rev. E, 84:017102, Jul 2011.

[17] Andrea Lancichinetti and Santo Fortunato. Benchmarks for testing community detection algorithms on directed and weighted graphs with overlapping communities. Physical Review E, 80(1):1-8, 2009.

[18] Andrea Lancichinetti and Santo Fortunato. Community detection algorithms: A comparative analysis. Phys. Rev. E, 80:056117, 2009.

[19] Andrea Lancichinetti and Santo Fortunato. Limits of modularity maximization in community detection. Phys. Rev. E, 84:066122, Dec 2011.

[20] Elizabeth A. Leicht and Mark E. J. Newman. Community structure in directed networks. Phys. Rev. Lett., 100(11):118703, 2008.

[21] Vincent Levorato and Coralie Petermann. Detection of communities in directed networks based on strongly p-connected components. In International Conference on Computational Aspects of Social Networks (CASoN), IEEE, pages 211-216, oct. 2011.

[22] Robert J. Mokken. Cliques, clubs and clans. Quality \& Quantity, 13(2):161-173, April 1979.

[23] Gergely Palla, Imre Derényi, Illés Farkas, and Tamás Vicsek. Uncovering the overlapping community structure of complex networks in nature and society. Nature, 435:814-818, June 2005.

[24] Gergely Palla, Illés J Farkas, Péter Pollner, Imre Derényi, and Tamás Vicsek. Directed network modules. New Journal of Physics, 9(6):186, 2007.

[25] Andrew Rosenberg and Julia Hirschberg. V-measure: A conditional entropy-based external cluster evaluation measure. In Joint Conference on Empirical Methods in Natural Language Processing and Computational Natural Language Learning (EMNLP-CoNLL), pages 410-420, 2007.

[26] Martin Rosvall and Carl T. Bergstrom. Maps of random walks on complex networks reveal community structure. Proceedings of the National Academy of Sciences USA, pages 11181123, 2008. 
[27] Philipp Schuetz and Amedeo Caflisch. Efficient modularity optimization by multistep greedy algorithm and vertex mover refinement. Phys. Rev. E, 77:046112, Apr 2008.

[28] Prithviraj Sen, Galileo Mark Namata, Mustafa Bilgic, Lise Getoor, Brian Gallagher, and Tina Eliassi-Rad. Collective classification in network data. AI Magazine, 29(3):93-106, 2008.

[29] Robert E. Tarjan. Depth-first search and linear graph algorithms. SIAM Journal on Computing, 1(2):146-160, 1972.

[30] Robert E. Tarjan. Enumeration of the Elementary Circuits of a Directed Graph. SIAM Journal on Computing, 2(3):211-216, 1973.

[31] Stéphan Thomassé. A quadratic kernel for feedback vertex set. In Proceedings of the twentieth Annual ACM-SIAM Symposium on Discrete Algorithms, SODA '09, pages 115119, Philadelphia, PA, USA, 2009. Society for Industrial and Applied Mathematics.

[32] Stijn Van Dongen. Graph clustering via a discrete uncoupling process. SIAM. J. Matrix Anal. \& Appl., 30(1):121-141, 2008.

[33] Stanley Wasserman and Katherine Faust. Social Network Analysis: Methods and Applications. Cambridge University Press, 1994.

[34] Fang Wu and Bernardo A. Huberman. Finding communities in linear time : A physics approach. The European Physical Journal B Condensed Matter, 38(2):331-338, 2003.

[35] Tianbao Yang, Yun Chi, Shenghuo Zhu, and Rong Jin. Directed network community detection: A popularity and productivity link model. In SDM'10: Proceedings of the 2010 SIAM International Conference On Data Mining, 2010. 